\title{
Epidemiology of Ovine Pasteurellosis in Lume District, East Shewa Zone of Oromiya Region, Ethiopia
}

\author{
Sadia $\mathrm{H}^{1}$, Abunna $\mathrm{F}^{2}$ and Jarso $\mathrm{D}^{2 *}$ \\ ${ }^{1}$ Oromia Livestock and Fishery Resource Development Bureau, Addis Ababa, Ethiopia \\ ${ }^{2}$ Department of Veterinary Medicine and Agriculture, Addis Ababa University, Ethiopia
}

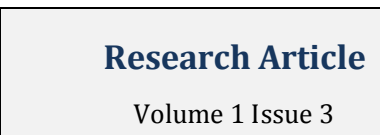

Received Date: July 13, 2016

Published Date: October 07, 2016

*Corresponding author: Jarso D, Department of Veterinary Medicine and Agriculture, Addis Ababa University, Ethiopia, E-mail: djmojo74.ethio@mail.com

\section{Abstract}

The study of ovine pasteurellosis was conducted in Lume districts, East Shoa Zone of Oromia region, Ethiopia to determine the prevalence of Mannheimia haemolytica, Pasteurella trehalosi and Pasteurella multocida from nasal swabs (384), abattoir specimens (145), and the serotype diversity among the species from sheep sera (150). A total of 115 isolates of M. haemolytica, P. trehalosi and P. multocida were isolated from nasal swabs of apparently health and clinically sick sheep and from pneumonic lungs. The M. haemolytica, P. trehalosi and Pasteurella multocida were isolated from the nasal swabs (11.2\%), (7.6\%) and (2.1) respectively, whereas M. haemolytica and P. trehalosi were isolated from pneumonic lungs (11.7\%), (10.3) respectively. However, Pasteurella multocida was the lowest among species isolated (2.1\%). The overall isolation rate of M. haemolytica, P. trehalosi and Pasteurella multocida was $15.7 \%, 11.5 \%$ and $2.9 \%$, respectively. From 145 lung samples collected and cultured, Pasteurella was isolated successfully in 35 (24.1\%) sheep. Out of 35 sheep lung lesion sample, the percentage recovery rate of M. haemolytica 17(11.7\%), P. trehalose 15(10.3\%) and P. multocida 3(2.1) \% respectively. On the basis of these results, M. haemolytica and P.trehalose were the most common cause of pasteurellosis in sheep at the study area. A total of 150 sheep sera were examined for serotype specific antibodies using indirect haemagglutination test for M. haemolytica, P. trehalose and P. multocida serotypes. Variation in prevalence among the different serotypes was observed $(\mathrm{P}<0.001)$. The IHA test revealed that serotype A1, A2, A7, T3, $\mathrm{T} 10$, and T15 were the dominant serotypes with $23.3 \%, 42.6 \%, 32,51.3,29.3$ and $30 \%$ positive by IHA whereas serotypes P. multocida biotype A and T4 were the least positive with $14.6 \%$ and $16 \%$ respectively. Generally, both bacterial and serological results of this study revealed that the causal agents of pasteurellosis are prevalent in the area, and serotypes A1, A2, A7, T3, T10 and T15 were dominant over the other serotypes.

Keywords: Mannheimia and Pasteurella spp; Nasal swabs; Isolation; Serotypes; Ethiopia

\section{Introduction}

Ovine pasteurellosis is disease mainly caused by Mannhaemia haemolytica (Pastuerella hemolytica),
Pastuerella multocida and Pastuerella trehalosi (Bibersteinia trehalosi) are the three most commonly isolated bacterial agents from pneumonias that result in high rates of morbidity and mortality in sheep. Pasteurella 


\section{Open Access Journal of Veterinary Science \& Research}

multocida and Mannheimia haemolytica are causative agents of several economically significant veterinary diseases. Serious infectious diseases as fowl cholera, bovine hemorrhagic septicemia, and porcine atrophic rhinitis are caused by $P$. multocida whereas $M$. haemolytica is the causative agent of shipping fever or pneumonic pasteurellosis in sheep [1].

In Ethiopia, a number of attempts have been made so far to identify the different serotypes of both Mannheimia and Pasteurella spp. in small ruminants. Recent work on pasteurellosis of small ruminants has demonstrated different species and serotypes of, M. haemolytica and $P$. trehalosi such as A1, A2, A5, A6, A7, A8, A9, A11, A12, A13, A14 and T3, T4, T10 and T15. Of which A1, A2, A8, A7, T3 and $\mathrm{T} 4$ are the dominant serotypes [2-5]. Small ruminant production in the study area is playing an important role in generating cash income, manure, social value, meat, skin. The predominant practices are mixed management system, traditional housing and grazing of natural pasture. Several investigations have been conducted in different countries and regions on the species and its serotypes that cause Pasteurellosis in sheep.

The different studies conducted in Ethiopia indicated that pasteurellosis is a major threat to sheep production. Some of these studies were those in Amhara Regional State particularly Debre Birhan [6] and South Wollo [7] and Oromia Regional State particularly selected sites of Arsi Zone [5] as well as those in Debre Birhan, Harshin and Jijiga but there is no available study in East Shoa Zone of Oromia region. In Lume district, despite annual vaccination against pneumonic pasteurellosis with a monovalent vaccine (inactivated P. multocida biotype A), there are high mortality and morbidity following respiratory distress (OBOARDAR, 2012/2013) [8]. Pasteurollosis is therefore a high-priority issue at the national level due to the significant economic losses it causes through mortality, morbidity, and the high cost of treatment. The main problem is absence of an extensive study on epidemiology of this disease as well as absence of a cost effective prevention and control methods which suits best for different phenotypes and serotypes of the agent. Therefore, the objectives of this study are to isolate and characterize most prevalent Pasteurella and Mannheimia species in sheep and to determine the possible major serotypes of Pasteurella involved in respiratory symptoms/infections of sheep in Lume districts of East Shoa zone of the Oromia region.

Jarso D. Epidemiology of Ovine Pasteurellosis in Lume District, East Shewa Zone of Oromiya Region, Ethiopia. J Vet Sci Res 2016, 1(3): 000115.

\section{Materials and Methods}

\section{The study area}

The study was conducted in Oromia Region; East shewa Zone at Lume district. The area is located at $74 \mathrm{~km}$ from Addis Ababa and $27 \mathrm{~km}$ from Bishoftu, at Longitude between 3856 $6^{\prime} \mathrm{E}-39^{\circ}{ }^{\circ} 17^{\prime} \mathrm{E}$ and Latitude $8^{\circ} 34^{\prime} \mathrm{N}-8^{\circ}{ }^{\circ} 34^{\prime} \mathrm{N}$. The average Elevation of the area is $1780 \mathrm{~m}$ above sea level. The area have average annual rain fall $969.35 \mathrm{~mm}$, and mean annual temperature of $20.4^{\circ} \mathrm{C}$. Management system of the animals is extensive production system. The main farming system is mixed farming and sheep are the predominant animal species kept in the area. Traditional housing and grazing of natural pasture are the predominant husbandry practices (OBoARDAR, 2012/2013) [8]. The district was selected due to having high population of small ruminants, high reports of the pasteurollosis disease challenge and it also the schematic area of the sponsorship.

\section{Study animals}

For the determination of contributing factors to epidemiology of ovine Pasteurellosis, indigenous sheep breeds belonging all age, sex and health status kept under extensive management system were sampled. The study animals were clinically healthy and sick sheep from five veterinary clinics for nasal swab and serum sampling and apparently healthy and slaughtered sheep from hotels were visited during sample collection. Accordingly 384 nasal swabs, 145 lung lesions and 150 serum samples were collected from the study area.

\section{Study design}

The type of the study was cross-sectional, with simple random sampling technique which was conducted from October 20014-March 20015 to establish the prevalence of Pasteurella trehalosi, Pasteurella multocida and Mannheimia haemolytica isolated from nasal swab of apparently healthy and sick sheep ,lung lesion from privately owned hotels in Lume district using isolation rates on culture and biochemical tests to identify their distribution among different age groups, sex, and health status and to determine the serotype prevalence of Mannheimia haemolytica P. trehalosi and P. multocida from serum samples of sheep collected in the study district.

The study PAs were selected by purposive sampling technique based on sheep population within PAs. The number of sheep to be sampled from each PAs of the 
districts was determined simply by equally dividing the share of the PAs and finally individual sheep sample was selected purposely from each PAs and then bacterial samples were collected from each sample individual at time of visit. Since the prevalence of ovine pasteurellosis in the selected district were not known, the sample size were determined using the formula given by Thrusfield [9] based on maximum expected prevalence of $50 \%$.

\section{Sample collection and laboratory analysis}

Nasal swabs were collected from randomly selected sheep. Before collecting the swabs, the nostrils of the animals were well cleaned with cotton wool soaked in $70 \%$ ethyl alcohol. Then sterile cotton-tipped swabs in screw-capped test tube moistened with tryptose soya broth (Oxoid, Hampshire, England) were inserted into the nostrils of each sheep, and the mucosa surface rubbed by rotating the swabs. The swabs were then placed back into $3 \mathrm{ml}$ of sterile tryptose soya broth (Oxoid, Hampshire, England) in universal tubes and transported packed in ice to the laboratory after collection and incubated immediately at $37^{\circ} \mathrm{C}$ for 24 hours. Lung lesions were collected from randomly selected slaughtered sheep at Lume privately owned hotels. Piece of affected part of lung of the corresponding animals were taken after close inspection and put into separate sterile containers and transported to the laboratory in cool box [10].

Blood samples for serum extraction were collected directly from jugular vein using sterile needles and plain vacuntainer tubes from randomly selected sheep flock. Up to $5-8 \mathrm{ml}$ of blood was withdrawn and the tubes left to stand in inclined position over night at an ambient temperature to allow clotting, and the sera were collected using sterile Pasture pipette and transferred to sterile testes tubes, labeled and stored at $-20^{\circ} \mathrm{C}$ in deep freezer.. Whereas lung samples were disinfected with $70 \%$ alcohol and dried, and the samples were cut into pieces with sterile scissors assisted by tongue forceps and put into 3 $\mathrm{ml}$ of tryptose Soya broth (Oxoid, Hampshire, England) in universal tubes. The universal tubes were loose capped and incubated at $37^{\circ} \mathrm{C}$ for 24 hours. After 24 hours incubation of the nasal swabs, lung tissues samples in tryptose Soya broth (Oxoid, Hampshire, England), a loop full of culture was transferred onto blood agar (Titan Biotech Limited, Bhiwadi) containing 7\% difibrinated sheep blood and MacConkeys agar (Titan Biotech Limited, Bhiwadi), and then streaked by inoculating loop. The plates were incubated at $37^{\circ} \mathrm{C}$ for 24 hours, and after 24 hours incubation, blood agar plates examined for the presence and type or absence of haemolysis and general appearance of the colonies including colour, shape, size and contour. The colonies suggestive of Pasteurella and Mannheimia were selected and smears were stained with Gram's staining and microscopically examined under oil immersion. Gram-negative, coccobacilli, short rods with or without bipolar staining were subjected for oxidase, urease, catalase, indole and $\mathrm{H}_{2} \mathrm{~S}$ production tests. Colonies, which were oxidase positive, urease negative, indole negative/positive, catalase positive/negative and yellow (acid) slant, yellow (acid) butt, H2S negative in TSI agar were subjected for further biochemical tests.

The growth on MacConkeys agar (Titan Biotech Limited, Bhiwadi) was examined for the presence or absence of growth and lactose fermentation as indicated by pink coloured colonies and non-lactose fermentation by absence of pink colour and general appearance of colonies. Accordingly colonies were grouped as lactose fermenters (LF) and non-lactose fermenters (NLF). Plates with Pasteurella and Mannheimia like colonies were kept for further biochemical testing whereas mixed colonies were further sub-cultured.

After primary characterization and oxidase, urease, indole, catalase and $\mathrm{H}_{2} \mathrm{~S}$ test of the isolates, biochemical characterization of the organisms was preformed based on Glucose, lactose, maltose, salicin, sucrose, trehalose and xylose and nitrate reduction tests. Mannheimia haemolytica isolates were selected on the basis of xylose and lactose fermentation and lack of fermentation of trehalose and salicin and were catalase positive. Whereas, isolates of Pasteurella trehalosi utilised only trehalose and salicin and were catalase negative. Pasteurella multocida were selected on the basis of indole production, nitrate reduction, characteristics sweetish odour and absence of growth and haemolysis on MacConkey and blood agar, respectively.

Sera were serotyped for $M$. haemolytica using the indirect haemagglutination (IHA) test introduces by Biberstein (1978) for serotyping Mannheimia haemolytica. Serotping was conducted using capsular extract antigen. Briefly, Capsular antigen was extracted from a $24 \mathrm{hr}$ culture of bacteria of known serotypes in tryptose Soya broth, which was inactivated in a water bath at $60^{\circ} \mathrm{C}$ for 30 minutes, and centrifuged at $3000 \mathrm{rpm}$ for 30 minutes; the clear supernatant was collected into sterile test tubes to be used as capsular extract antigen. Fresh sheep blood was collected in Alsever"s solution at proportion of 3:5. The suspension was centrifuged at $2500 \mathrm{rpm}$ for 5 minutes, washed twice with phosphate 
buffer saline solution (PBSS), and again centrifuged at $2500 \mathrm{rpm}$ for 5 minutes. For sensitization of the sheep red blood cells (RBC), $50 \mu$ l of packed RBC were added to $5 \mathrm{ml}$ of capsular extract antigen, and then $50 \mu \mathrm{l}$ of glutaraldehyde was added and homogenized with gentle shaking, incubated for $1 \mathrm{hr}$ at $37^{\circ} \mathrm{C}$. After incubation the suspension was centrifuged and washed twice with PBSS. Finally, the pellet was adjusted with PBSS to give a1\% suspension of RBC. In $v$ bottomed micro-plates $50 \mu$ l of PBSS were added to all wells and $50 \mu \mathrm{l}$ of test sera to the first column and serially diluted by pipetting $50 \mu \mathrm{l}$ up to column 12. Fifty micro liters of sensitized RBC were added to each well and incubated for one hour at $37^{\circ} \mathrm{C}$. Results were recorded based on complete or more than $50 \%$ agglutination seen in each well. The titer showing $1 / 20$ dilution and above were taken as positive.

\section{Data analysis}

For interpretation of the results, after entry of the collected data into the Microsoft Excel sheet, it was summarized by descriptive statistics and then displayed by tables and graphs to illustrate the relationships between the dependent variables (each Pasteurella species and their total) and independent variables (PAs, age, sex and health status). Chi-square $\left(x^{2}\right)$ tests for repeated measures were used to test relationship between dependent variable (Pasteurella species distribution) and different independent host and environmental factors. For these analyses SPSS statistics 20 and Epi Info were used. Prevalence of Pasteurella and Mannheimia isolates were analyzed using percentages and serotype distribution compared using percentages and mean percentages.

\section{Results}

Pasteurella and Mannheimia isolates were revealed different morphological features on smear made from fresh isolate cultures. The isolates were Gram-negative, short ovoid rods with an occasional tendency to bipolar staining. Cells arranged in chain were also observed.

The Mannheimia haemolytica and Pasteurella trehalosi revealed moist, smooth greyish, odourless and haemolytic colonies on blood agar, while P. multocida revealed round, greyish, non-haemolytic occasionally mucoid colonies. All the isolates of Mannheimia haemolytica and pasteurella trehalosi were grown on MacConkey's agar and showed pink to red small pinpoint colonies. None of P. multocida isolates showed growth on MacConkey agar.

From 529 samples (384 nasal swabs and 145 lung lesion) collected and cultured, Pasteurella was isolated successfully in 115 sheep. Out of $115,80(21.1 \%)$ were from nasal cavities and 35(24.1\%) from lungs. The prevalence of $M$. haemolytica 43(11.2\%), P. trehalose $29(7.6 \%)$ and P. multocida $8(2.1 \%)$ from the nasal swab sample respectively. Whereas in lung lesion, of the samples which were culture positive, $17(11.7 \%)$ of the isolate was M. haemolytica and 15(10.3\%) of the isolate was $P$. trehalose and $3(2.1 \%)$ of the isolate was $P$. multocida. On the basis of these results $M$. haemolytica and $P$. trehalose was the most common cause of pasteurellosis in sheep at Lume district regardless of the health status, age, sex and other attributes (Table 1).

\begin{tabular}{|c|c|c|c|c|c|}
\hline \multirow{2}{*}{ Type-of sample } & \multirow{2}{*}{$\begin{array}{c}\text { No of sample } \\
\text { processed }\end{array}$} & \multirow{2}{*}{ Total positive } & \multicolumn{3}{|c|}{ Species identified } \\
\cline { 4 - 6 } & & & M. haemolytica & P. multocida & P. trehalosi \\
\hline Nasal swab & 384 & $80(21 \%)$ & $43(11.2 \%)$ & $8(2.1 \%)$ & $29(7.6 \%)$ \\
\hline $\begin{array}{c}\text { Lung lesion } \\
\text { sample }\end{array}$ & 145 & $35(24.1 \%)$ & $17(11.7 \%)$ & $3(2.1 \%)$ & $15(10.3 \%)$ \\
\hline Total & 529 & $115(21.7 \%)$ & $60(11.3 \%)$ & $11(2.1 \%)$ & $44(8.3 \%)$ \\
\hline
\end{tabular}

Table 1: Prevalence of M. haemolytica, P. trehalosi and P. multocida isolates of sheep.

From the total isolates Mannheimia haemolytica, $P$. trehalosi and P. multocida 43(11.2\%), 29(7.6\%) and 8 $(2.1 \%)$ positive in nasal swabs respectively. Out of these isolates, $21(9.3 \%)$ and $60(38.2 \%)$ were isolated from apparently healthy and clinically sick sheep with respiratory syndrome, respectively. There was high significant difference $(\mathrm{P}<0.001)$ between isolates from nasal swabs of healthy and clinically sick sheep with respiratory syndrome (Table 2 ). 


\section{Open Access Journal of Veterinary Science \& Research}

\begin{tabular}{|c|c|c|c|c|}
\hline Status of sheep & \multirow{2}{*}{ No (\%) } & \multicolumn{3}{|c|}{ Isolated species (\%) } \\
\cline { 3 - 5 } & & M. haemolytica & P. trehalosi & P. multocida \\
\hline Healthy sheep & $227(21)$ & $11(4.8)$ & $8(3.5)$ & $2(0.8)$ \\
\hline Diseased sheep & $157(59)$ & $32(20.3)$ & $21(13.3)$ & $6(3.8)$ \\
\hline Total & $384(80)$ & $43(11.2)$ & $29(7.5)$ & $8(2.08)$ \\
\hline
\end{tabular}

Table 2: The prevalence of M. haemolytica, P. trehalosi and P. multocida from apparently healthy and clinically sick sheep.

Regardless of species of the isolates and PAs the prevalence of the isolates in Qoka was $27.2 \%$, Sheran Dibandiba $14.2 \%$, Jido $19.4 \%$, Biyo $23.3 \%$ and in Mojo $21.1 \%$. These show there were variations in distribution of the agent among the five study PAs but the variation is not statistically significant because $\mathrm{p}=0.788$. Age distribution of the total positivity shows that the isolation rate of the agent varies significantly among different age groups $(\mathrm{P}=0.001)$.The isolation rate of the agent increases from age categories 1-3mth (15) to 4-6mth (16), from7$9 \mathrm{mth}(14)$ to 10-12mth (11) ,1-3yrs (9) and from4-6year (8) to 7-9yrs (11). Whereas Sex and distribution of agent has no statistical significant variation even if there were variations observed.

\begin{tabular}{|c|c|c|c|c|c|c|}
\hline \multirow{2}{*}{ Risk factors } & Level & No examined & No positive & Prevalence in \% & x2 & P-value \\
\hline PAs & Qoka & 77 & 21 & 27.2 & 1.17175 & 0.788 \\
\hline & Sh/dibandiba & 77 & 11 & 14.2 & & \\
\hline & Jido & 77 & 15 & 19.4 & & \\
\hline & Mojo & 76 & 16 & 21.1 & & \\
\hline Age & Biyo & 77 & 18 & 23.3 & & \\
\hline & <-3mth & 65 & 15 & 23.1 & 10.24 & 0.001 \\
\hline & $7-9 m$ mth & 57 & 16 & 22.8 & & \\
\hline & $10-12 m$ th & 54 & 11 & 17.3 & & \\
\hline & $1-3 y r s$ & 53 & 9 & 17.1 & & \\
\hline & $4-6 y r s$ & 47 & 8 & 17.1 & & \\
\hline & $7-9 y r s$ & 44 & 11 & 25 & & \\
\hline Sex & Female & 201 & 47 & 23.4 & 0.0296 & 0.863 \\
\hline & Male & 183 & 34 & 18.6 & & \\
\hline Health status & Healthy & 227 & 21 & 10.2 & 8.858 & 0.0001 \\
\hline & Sick & 157 & 59 & 38.2 & & \\
\hline
\end{tabular}

Table 3: Distribution of total positivity nasal swab among different epidemiological risk factors.

When the distribution of the agent by their species was seen among PAs, there were variations observed in distribution of a single species of the isolate among PAs. Even if variation is observed among PAs distribution of isolates was independent of PAs which means they have no statistically significant association between the isolates and PAs. Likewise age and sex distribution of isolates were examined for similarity in distribution of isolates among different age groups and sex of sheep. A Pearson chi-square test computed for existence of dependency between isolates and age of sheep shows that there was dependency between age of sheep and 
distribution of the disease agents which implies that at $95 \%$ confidence level distribution of isolates is dependent on age of sheep (P-value $<0.05$ ). That is statistically there is significant association between distribution of isolates and age of sheep. Sex isolate cross tabulation shows that there was no significant variation in distribution of isolates both within female and male sheep and also between female and male sheep. That is distribution of isolates was independent of sex of sheep; sex of sheep does not limits distribution of isolates. This implies that sex (host factor) is not a determinant factor of the disease pasteurellosis in sheep (Table 3).

Isolates and health status of sheep shows that there were dependencies between isolates and health status of sheep, indicating that distribution of isolates were dependent of health status of sheep. For the purpose of Mannheimia and Pasteurella isolation, lung lesion of sheep were examined from slaughtered sheep at voluntary hotels in which highest number of sheep slaughtered on daily basis were selected for three months, November, December and January during the study period. Sampling of the specimen was performed at a time.
From 145 lung samples collected and cultured, Pasteurella was isolated successfully in 35(23.4\%) sheep. Out of 35, the prevalence of M. haemolytica $17(11.7 \%), P$. trehalose 15(10.3\%) and P. multocida 3(2.1) \% from the lung lesion sample respectively. On the basis of these results $M$. haemolytica and $P$. trehalose was the most common cause of pasteurellosis in sheep at Lume district. However, the observed prevalence of isolates from lung in sex and age group has no impact on the isolation of pasteurella species.

Sheep Sera collected from Lume districts East shoa Zone of Oromia region, namely Qoka, Sheran dibandiba, Jido, Biyo and Mojo were serotyped using the indirect haemoagglutination (IHA) test as per Biberstein (1978) for serotyping of $M$. haemolytica, P.multocida and $P$. trehalosi. A total of 150 serum samples, thirty sera from each PAs were examined for specific antibodies. The $M$. haemolytica, $P$. multocida and $P$. trehalosi serotype positives have been computed for each PAs.

\begin{tabular}{|c|c|c|c|c|c|c|}
\hline \multirow{3}{*}{$\begin{array}{c}\text { Mannhaemia/Pasteurella } \\
\text { serotypes } \\
\end{array}$} & \multicolumn{6}{|c|}{ Seropositive (\%) at dilution $\geq 1 / 20$} \\
\hline & Jido & Qoka & Sh/dibandiba & Mojo & Biyo & \multirow{2}{*}{$\begin{array}{c}\text { Total No } \\
(\%)\end{array}$} \\
\hline & $\mathrm{No}=30$ & $\mathrm{No}=30$ & No $=30$ & $\mathrm{No}=30$ & $\mathrm{No}=30$ & \\
\hline A1 & $7(23.3)$ & $3(10)$ & $14(46.6)$ & $9(30)$ & $2(6.6)$ & $35(23.3)$ \\
\hline A2 & $17(56.6)$ & $15(50)$ & $11(36.6)$ & $9(30)$ & $13(43.3)$ & $65(43.3)$ \\
\hline A7 & $13(43.3)$ & $7(23.3)$ & $8(26.6)$ & $11(36.6)$ & $9(30)$ & $48(32)$ \\
\hline PA & $5(16.6)$ & $4(13.3)$ & $4(13.3)$ & $6(20)$ & $3(10)$ & $22(14.6)$ \\
\hline T3 & $18(40)$ & $17(56.6)$ & $15(50)$ & $16(53.3)$ & $11(36.6)$ & $77(51.3)$ \\
\hline $\mathrm{T} 4$ & $12(40)$ & $6(20)$ & $9(30)$ & $7(23.3)$ & $8(26.6)$ & $42(28)$ \\
\hline $\mathrm{T} 10$ & $8(26.6)$ & $11(36.6)$ & $7(23.3)$ & $9(30)$ & $9(30)$ & $44(29.3)$ \\
\hline T15 & $13(43.3)$ & $8(26.6)$ & $9(30)$ & $7(13.3)$ & $8(26.6)$ & $45(30)$ \\
\hline
\end{tabular}

Table 4: Prevalence distribution of Pasteurella serotypes in sheep sera in different PAs of Lume districts.

Variation in prevalence among the different serotypes was observed, in Mojo district, serotype A1 (30\%) followed by $\mathrm{A} 2(30 \%)$ and $\mathrm{A} 7(36.6 \%)$, $\mathrm{PA}(20 \%)$, T3(53.3\%), T4(23.3\%), $\mathrm{T} 10(30 \%)$ and $\mathrm{T} 15(23.3 \%)$. in Qoqaa A1(10\%), A2(50) and A7(23.3 \%) PA(13.3\%), T3(56.6\%), T4(20\%), T10(36.6\%) and T15(26.6\%) each in Sheran Dibandiba A1(46.6\%), A2(36.6 \%), A7(26.6\%), $\mathrm{PA}(13.3 \%), \quad \mathrm{T} 3(50 \%), \quad \mathrm{T} 4(30 \%), \quad \mathrm{T} 10(23.3 \%) \quad$ and T15(30\%). In Jidoo A1 (23.3\%), A2 (56.6\%) and A7
(43.3\%), PA (16.6\%), T3 (40\%), T4 (40\%), T10(26.6\%) and $\mathrm{T} 15(43.3 \%)$ and in Biyo, $\mathrm{A} 1(6.6 \%), \mathrm{A} 2(40 \%)$, A7(30\%), PA(10\%), T3(36.6\%), T4(26.6\%), T10(30\%) and $\mathrm{T} 15(26.6 \%)$ were the dominant serotypes detected (Table 4).

In present study, there was higher prevalence of $M$. haemolytica A1 (23.3\%), A2(42.6\%), A7(32\%), T3(51.3), $\mathrm{T} 10(29.3)$ and $\mathrm{T} 15(30 \%)$ serotypes were lower 
prevalence of P.multocida biotype A(14.6\%) and T4(16\%) were recorded in the study areas (Table 5).

\begin{tabular}{|c|c|}
\hline $\begin{array}{c}\text { Sero-types of ovine } \\
\text { Total }\end{array}$ & $\begin{array}{c}\text { Prevalence } \\
\%\end{array}$ \\
\cline { 1 - 2 } pasteurollosis & $23.30 \%$ \\
\hline M.hemolytica A1 35 & $42.60 \%$ \\
\hline M.hemolytica A2 64 & $32 \%$ \\
\hline M.hemolytica A7 48 & $14.60 \%$ \\
\hline P.multocida biotype A 22 & $51.30 \%$ \\
\hline P.trehalosi T3 77 & $16 \%$ \\
\hline P.trehalosi T4 24 & $29.30 \%$ \\
\hline P.trehalosi T10 44 & $30 \%$ \\
\hline P.trehalosi T15 45 & \\
\hline
\end{tabular}

Table 5: Overall Prevalence of Ovine Pasteurellosis.

\section{Discussion}

Pasteurellosis in sheep caused by M. haemolytica and $P$. trehalosi have posed health problem in most part of sheep breeding and rearing regions of Ethiopia due to the significant economic losses they causes through mortality, morbidity, and the high cost of treatment Gelagay, et al. [6]. In this study, an attempt was made to differentiate between M. haemolytica, P. trehalosi and P. multocida based on their growth on MacConkey agar, haemolytic pattern, Oxidase and Catalase activity, Indole and $\mathrm{H}_{2} \mathrm{~S}$ production, colony morphology and fermentation of different sugars with acid production Quinn et al. [11]. The aetiological agent of pasteurellosis in sheep was found to be wide spreading in the study area. Though there were little variation in biochemical characteristics, the results of morphological, staining, colony, cultural and biochemical activities were in total agreement with those documented by Carter [12] and Quinn et al. [11]. The haemolytic activity of $M$. haemolytica and P. trehalosi isolates were lost after subsequent subcultures and were in agreement as reported by Quinn et al. [11]. Two distinct colony types were observed on MacConkey agar, lactose fermenter ( $M$. haemolytica) with pink and nonlactose fermenter ( $P$. trehalosi) other than pink colour. Whereas the $P$. multocida isolates neither grew on MacConkey agar nor haemolysed sheep red blood cells as observed by Quinn et al. [11]. The isolates of $M$. haemolytica, P. trehalosi and P. multocida were positive for oxidase test and negative for urease, HS2 production in TSI agar slant, and Indole test. Further they utilized glucose fermentatively, but the $P$. multocida isolates were indole positive which coincided with those described by Quinn et al. [11]. The Mannheimia haemolytica isolates fermented xylose and lactose, but failed to ferment trehalose and salicin, and were catalase positive, whereas isolates of $P$. trehalosi were fermented. Trehalose and salicin, and were catalase negative the results are in agreement with Carter [12] and Quinn et al. [12].

In the present study, M. haemolytica and P. trehalosi were isolated at the rate of $11.2 \%$ and $7.6 \%$ from nasal swabs, $11.7 \%$ and $10.3 \%$ from pneumonic lungs, respectively. Isolation of $M$. haemolytica and P. trehalosi from nasal swab and pneumonic lungs may indicate that these species are important species in the induction of pneumonic pasteurellosis in the study area.

The low percent of $(2.1 \%)$ both from nasal swab and lung lesion P. multocida isolates might indicate the occasional involvement of this species in the pneumonic pasteurellosis and similar reports have been made by Aschalew, Mekonen [5], Sisay and Zerihun [10] and Belay [7]. In contrary to our observation Assefa et al. and Mesele reported high incidence rates of $15.4 \%$ and $25 \%$ of $P$. multocida from sheep slaughtered at Jijiga and ELFORA abattoir respectively. This might be due to the time of sampling and geographical variation, where sampling was conducted in June and sampled sheep were from Somali lowlands where very poor Veterinary infrastructure and vaccination against pasteurollosis ( $P$. multocida biotype A) was not conducted for considerable number of years, and animals were transported long distance before being slaughtered at both abattoir, whereas, our study was conducted, where regular vaccination was done in sheep with monovalent $P$. multocida vaccine which is attributed to the low incidence of infection.

From total isolates of nasal swabs of clinically sick sheep with respiratory syndrome, $M$. haemolytica and $P$. trehalosi constituted $20.3 \%$ and $13.3 \%$ in their proportion, respectively. This result is in agreement with the findings of Tesfaye and Aschalew in highlands of central Ethiopia, where they reported $28.8 \%$ and $35 \%$ for M. haemolytica and $42 \%$ and $58.3 \%$ for P. trehalosi, respectively from nasal swabs of sheep with pneumonic symptoms. The high proportion of Mannheimia and Pasteurella species in nasal swabs of clinically sick sheep could be due to these organisms are normal inhabitants of the upper respiratory tract of sheep and invasion to the lower tissue (lung) of clinically sick sheep when the 
immune system of the animals is compromised by different factors [13] and pneumonia associated with these bacteria species recorded as primary diseases in sheep elsewhere.

Accordingly, there was a significant difference existing among isolation rates at different time in different areas even if there is no significant difference in isolation rates among the three species in all of the studies at different time and areas. This implies that there must be a continuous survey to be held in different areas to know a recent rate for each of the species implicated for ovine pasteurellosis and to design a cost effective and efficient prevention and control strategies suited for each area.

Age distribution of the three species associated with ovine pneumonic pasteurellosis disclose that there was an association in distribution of the species among different age groups of sheep and at the same time distribution of the species was also associated with age groups of sheep. According to the present study different age group distribution of the isolates looked like as follows: within age group $1-3 \mathrm{mth}$ it was $23.1 \%$, 4-6mth $(22.8 \%), 7-9 \mathrm{mth}$ (22.2\%), 10-12mth (20.3\%), 1-3year (17.1\%), 4-6year $(17.1 \%)$ and $7-9$ year it was (25\%). This was in agreement with that of the result obtained by Behailu. This result ease also in agreement with findings of Gilmour and Gilmour [5], that elucidates pneumonic pasteurellosis occur in all ages of sheep and goats, with the most susceptible in lambs and kids during first life, and dams at lambing but not agreement with Zuber [19] in Iraq with slight fluctuation in age grouping which was in $1-3 \mathrm{mth}$ age category it was $2.1 \%, 4-8 \mathrm{mth}(7.7 \%)$ and 9 $12 \mathrm{~m}$ th $(7.1 \%)$.

Sex shows that there was no significant variation in distribution of the species between female and male sex and that the distribution was independent of sex of sheep. In the present study prevalence of the agent in female sheep was $23.4 \%$ while it was $18.6 \%$ in male. This result was in agreement with Behailu where female $29.2 \%$ and male $24.6 \%$, and also with Belay [7].

The prevalence of $M$. haemolytica, P.trehalosi and P.multocida was almost similar in nasal swabs $(11.2 \%)$, (7.6\%) and (2.1\%) and in lung sample (11.7\%), (10.3\%) and (2.1\%) respectively. In this study, M. haemolytica and $P$. trehalosi in pneumonic lungs may indicate that these species are important species in the induction of pneumonic pasteurellosis in the study area. The low percent of $(2.1 \%)$ P. multocida from lung lesion isolates might indicate the occasional involvement of this species in the pneumonic pasteurellosis. No associations were observed between the risk factors and pneumonic pasteurellosis at lung lesion sample $(P>0.05)$. This study result is in agreement with the reports of Marru, $M$. haemolytica (21.96\%) and absolute prevalence of $P$. multocida and Kaoud prevalence of 52\% for Mannheimia and $42 \%$ for $P$. trehalosi.

The serological results showed that the predominance distribution pattern of $M$. haemolytica and P. trehalosi serotypes between the PAs of Lume districts were almost similar. This might be due to similar agro climatic and animal management systems.

Of 150 sheep sera tested, A1(60\%), A2(42.6\%), A7(32\%) and PA(14.6\%), T3(51.3\%), T4(16\%), $\mathrm{T} 10(29.3)$ and $\mathrm{T} 15(30 \%)$ were detected. Of which, A1(60\%), A2(42.6\%), A7(32\%), PA(14.6\%), T3(51.3\%), $\mathrm{T} 10(29.3 \%)$ and $\mathrm{T} 15(30 \%)$ were the dominant serotypes and P.multocida biotype A were the least detected serotypes. The result of this study is in agreement with the reports of Yeshwas Ferede [16] P.haemolytica A1(33.1\%), A2(28.5\%) and A7(31.8\%) serotypes where as a lower prevalence of $P$. multocida biotype A (6.6\%) were recorded in South Gonder Zone Farta and Lay Gayint districts, the result this study was also in agreement with that of Megersa and Sisay and Zerihun [10] who reported that $29 \%$ and $16.9 \%$ prevalence for A1, in East and Northeast Ethiopia, respectively, which was the most prevalent serotype.

Pegram et al. [17] was also identified in Ethiopia the most prevalent serotype was A2, while A14 and A9 were the least identified. Hussein and Mohammed [18] in Sudan identified the predominant A2 and A6 serotypes and A13 and A14 were among the lowest identified serotypes. Ayelet also identifies in central highland of Ethiopia A2 and A6 were the most dominant ones, while A8 was the least screened serotype and Kirkan and Kaya and Lhan and Keles [19] in Turkey identified A2 and A6 as most prevalent and A8 was the least identified respectively, which was the most prevalent serotype. This deference in dominance of serotype might be due to geographical location, the time of sampling, serotypes involved in pasteurellosis which varied from year to year, area to area and flock to flock [14].

In conclusion, both bacterial and serological results of this study revealed that pasteurellosis is the most prevalent disease and serotypes A1, A2, A7, T3, T10 and 


\section{Open Access Journal of Veterinary Science \& Research}

T15 were dominant serotypes. Therefore, developing a polyvalent vaccine consisting of the most predominant serotypes of Mannheimia haemolytica which are circulating in sheep in the study area is of paramount importance

\section{References}

1. Michael W (2008) Pasteurellosis Transmission Risks between Domestic and Wild Sheep. CAST Commentary Pp. 325.

2. Gelagay A (1996) Epidemiology and serological investigation on mutifactorial ovine respiratory disease and vaccine trials on the highlands of north shoa (Ethiopia) DVM thesis. Addis Ababa University, Faculty of Veterinary Medicine, Debrezeit.

3. Tesfaye M (1997) Serological and bacterial investigation of P.haemolytica serotypes in sheep in high land of Wollo (North east Ethiopia), DVM thesis, Addis Ababa, FVM, Debrezeit.

4. Zeleke A (1998) A study of ovine pneumonic pasteurellosis in North Shoa. DVM Thesis, Faculty of Veterinary Medicine, Addis Ababa University, Debre Zeit, Ethiopia.

5. Mokonnen T (2000) An Epidemiological study on Ovine Pasteurollosis Arsi, South east Ethiopia. DVM thesis, AAU, FVM. Debrezeit.

6. Ayelet G, Laekemariam Y, Esayas G, Selam T, Kassahun A (2004) Epidemiologic and Serologic Investigation of Multifactorial Respiratory Disease of Sheep in the Central Highland of Ethiopia. Intern J Appl Res Vet Med 2(4): 274-278.

7. Belay M (2007) Study of Ovine Pasteurollosis in Wollo Zone of Amhara region, Ethiopia. DVM thesis, Addis Ababa, FVM, Debrezeit.

8. Oboardar (2013) Oromia Bureo of Agriculture and Rural Development Annual.

9. Thrusfield M V (1995) Veterinary Epidemiology, Black Wall Science, Great Britain, Pp: 183.

10. Sisay T, Zerihun A (2003) Diversity of M. haemolytica and P. treholsi serotypes from apparent healthy sheep and abattoir specimens in the high land of Wollo, North East Ethiopia. Vet Reserch 27(1): 3-14.
11. Quinn PJ, Morkey BK, Carter ME, Donnelly WJC, Leonard FC, et al. (2002) Pasteurella species and Mannheimia haemolytica. In: Veterinary Microbiology and Microbial Disease. Blackwell publishing company: Blackwell science. London Pp: 137-142.

12. Carter G R (1984) Pasteurella, Yesinia and Francisella. In: Charles C (Eds) Diagnostic Procedures in Veterinary Bacteriology and Mycology. Thomas publishing USA Pp 111-118.

13. Lopez A (2001) Respiratory system, thoracic cavity and pleura. In: M D McGavin, W W Carlton, J Zachary (Eds.) Thomson's Special Veterinary Pathology, Mosby-Year Book Inc Pp: 125-195.

14. Gilmour NJL, Gilmour JS (1989) Pasteurellosis of sheep. In: Adlam CF, Rutter JM (Eds) Pasteurella and Pasteurellosis. Academic Press Inc, New York, Pp: 223-262.

15. Zuber I (2009) Epidemiological Study of Pasteurellosis in Lambs in Erbil Governorate, Northern Iraq. UDC, 619: 579-843.

16. Yeshwas F, Shigdaf M, Hailu M, Agraw A (2013) Serotyping and evaluation of the level of protective antibody titer in northwest Ethiopian sheep before and after ovine Pasteurollosis vaccination. Int J Pharm Med \& Bio Sc 2(4): 1-8.

17. Pegram RG, PL Roeder, JM Scott (1980) The prevalence of serotypes of Pasteurella haemolytica in Ethiopia. Ethiop Vet Bull 4: 18-25.

18. Hussein A M, Elsawi Mohamed O A (1984) Serological survey of sheep sera for antibodies to Pasteurella haemolytica serotypes in Sudan. Rev Elev Med Vet Pays Trop 37(4): 418-421.

19. Lhan Z, Keles H (2007) Biotyping and serotyping of Mannheimia (Pasteurella) haemolytica isolated from lung samples of slaughtered sheep in the Van Region. Turk J Vet Anim Sci 31(2): 1-5. 